University of Nebraska - Lincoln

DigitalCommons@University of Nebraska - Lincoln

Faculty Publications from Nebraska Center for Materials and Nanoscience, Nebraska Center Materials and Nanoscience

for (NCMN)

8-1-2005

\title{
Atomic and micromagnetic aspects of L10 magnetism
}

\author{
Ralph Skomski \\ University of Nebraska-Lincoln, rskomski2@unl.edu \\ Arti Kashyap \\ University of Nebraska-Lincoln, akashyap@Inmiit.ac.in \\ Jian Zhou \\ University of Nebraska - Lincoln
}

Follow this and additional works at: https://digitalcommons.unl.edu/cmrafacpub

Part of the Nanoscience and Nanotechnology Commons

Skomski, Ralph; Kashyap, Arti; and Zhou, Jian, "Atomic and micromagnetic aspects of L10 magnetism" (2005). Faculty Publications from Nebraska Center for Materials and Nanoscience. 5.

https://digitalcommons.unl.edu/cmrafacpub/5

This Article is brought to you for free and open access by the Materials and Nanoscience, Nebraska Center for (NCMN) at DigitalCommons@University of Nebraska - Lincoln. It has been accepted for inclusion in Faculty Publications from Nebraska Center for Materials and Nanoscience by an authorized administrator of DigitalCommons@University of Nebraska - Lincoln. 


\title{
Atomic and micromagnetic aspects of $\mathrm{L}_{0}$ magnetism
}

\author{
R. Skomski*, A. Kashyap*†, and J. Zhou* \\ * Department of Physics and Astronomy \\ Center for Materials Research and Analysis \\ University of Nebraska-Lincoln \\ $\dagger$ IFW Dresden \\ Helmholtzstrasse 20 \\ Lincoln, NE 68588-0111, USA \\ Dresden D-01069, Germany
}

Submitted November 2004; accepted 21 April 2005; available online 23 May 2005; published August 2005.

\begin{abstract}
Atomic and continuum effects in $\mathrm{L}_{0}$ magnets are investigated. Emphasis is on the competition between ferromagnetism, antiferromagnetism, and noncollinear order in both perfect and imperfect structures, and on the temperature dependence of the magnetic anisotropy. The applicability of micromagnetic and atomistic approaches depends on the length scales involved, but there is a broad range of phenomena where both can be used.
\end{abstract}

Keywords: Magnetic anisotropy, Magnetic order, Finite-temperature magnetism, Noncollinearity

\section{Introduction}

Since the discovery of $\mathrm{L}_{0}$-type PtFe and PtCo magnets by Graf and Kussmann [1] and Jellinghaus [2], respectively, this fascinating class of hard-magnetic materials has attracted continuing attention in various areas, including high-density magnetic recording [3], [4], [5], [6], [7], [8], [9] and [10]. Scientific interest has been fueled by the specific features of $\mathrm{L}_{0}$ magnets, such as the layered crystal structure, the two-sublattice nature of the magnetism, and the simultaneous involvement of $3 \mathrm{~d}$ and $4 \mathrm{~d} / 5 \mathrm{~d}$ electrons, and continuum [9] and atomistic models [11], micromagnetic simulations [12], first-principles calculations [13] and [14], and multiscale approaches [15] are now being used or developed to describe the new nanoscale materials.

Magnetic $\mathrm{L} 1_{0}$ or $\mathrm{CuAu}(\mathrm{I})$ compounds having the nominal structure MT consist of alternating layers of light or $3 \mathrm{~d}$ transition-metal atoms $(\mathrm{T})$ and heavy or $4 \mathrm{~d} / 5 \mathrm{~d}$ transition-metal atoms (M), but the most general $\mathrm{L1}_{0}$ composition is $\mathrm{ABC}_{2}$, where $\mathrm{C}$ planes are separated by $\mathrm{A}-\mathrm{B}$ planes. Fig. 1 shows how the A, B and C atoms occupy the 1a, 1c and $2 \mathrm{e}$ sites, respectively. The site occupancy sites matters, because $3 \mathrm{~d}$ and $4 \mathrm{~d} / 5 \mathrm{~d}$ substitutions are useful to tune the Curie temperature $T_{\mathrm{c}}$, magnetization $M_{\mathrm{s}}$, and magnetocrystalline anisotropy $K_{1}$ of magnetic recording materials, and because the control of $\mathrm{L} 1_{0}$ order is a generally quite demanding task.

From a theoretical point of view, first-principles calculations are now able to predict moments [16] and [17], bulk and thin-film anisotropies [18], site-resolved exchange interactions [19], exchange stiffnesses [20], and Curie temperatures [6] and [14] for ferromagnetic and simple antiferromagnetic structures [19] and [21]. However, there is a broad and largely unexplored range of competing antiferromagnetic and noncollinear spin structures. From a macroscopic starting point, continuum or 'micromagnetic' approaches describe magnetization processes such as the nucleation of reverse domains and domain-wall motion [9] and [12], but the question arises whether these approaches are able to account for phenomena such as domain wall pinning at antiphase boundaries, nanoparticle surface anisotropy, and fast switching dynamics.

In this paper, we discuss zero-temperature and finitetemperature effects in $\mathrm{L1}_{0}$ magnets and discuss the involved length and energy scales. Section 2 deals with spin structure in the absence of spin-orbit coupling, Section 3 investigates mechanisms of noncollinearity, and Section 4 deals with finite-temperature anisotropy. Finally, Section 5 compares the applicability of atomistic and continuum approaches. 


\section{Atomic-scale spin structure}

To large extent, the spin structure of $\mathrm{L}_{0}$ magnets is determined by Heisenberg exchange, $J\left(\mathbf{R}_{i}-\mathbf{R}_{j}\right) \mathbf{S}_{i} \cdot \mathbf{S}_{j}=J_{i j} \mathbf{S}_{i} \cdot \mathbf{S}$ $j$, a relatively strong interaction of electrostatic origin. It is magnetically isotropic, meaning that uniform spin rotation does not change the magnetic energy. For example, intraand interlayer interactions in $\mathrm{L}_{0}$ magnets exhibit a bond anisotropy [22], but the corresponding energy is independent of angle between magnetization and $c$-axis. Depending on band filling and interatomic distance, Heisenberg exchange in metals may be ferromagnetic $\left(J_{i j}>0\right)$ or antiferromagnetic $\left(J_{i j}<0\right)$. A simple and asymptotically correct [23] and [24] model is the Ruderman-Kittel-Kasuya-Yosida or RKKY exchange, $J_{i j}=J\left(R_{i j}\right) \sim \cos \left(2 k_{\mathrm{F}} R_{i j}\right) / R_{i j}^{3}$. Pictorially, a localized spin in an electron gas behaves like a stone thrown into water, and the minima and maxima of the resulting wave are analogous to the RKKY oscillations. The simultaneous presence of ferro- and antiferromagnetic interactions leads to a competition between various types of magnetic order, including ferromagnetism, different types of antiferromagnetism, and noncollinear spin structures. In a addition, some or all atoms may be Pauli paramagnetic. Fig. 2 shows some spin structures that exist or may exist in $\mathrm{L}_{0}$ magnets.

Elemental $4 \mathrm{~d} / 5 \mathrm{~d}$ magnets, such as $\mathrm{Pd}$ and $\mathrm{Pt}$, are exchanged-enhanced Pauli paramagnets, but in a ferromagnetic environment they are easily spin-polarized by neighboring $3 \mathrm{~d}$ atoms. The $4 \mathrm{~d} / 5 \mathrm{~d}$ moment contributes little to the magnetization and Curie temperature, but it plays a key role in the realization of magnetic anisotropy (Section 4). In antiferromagnets, the $4 \mathrm{~d} / 5 \mathrm{~d}$ moment is zero, because the polarization contributions of neighboring $3 \mathrm{~d}$ atoms cancel each other, as illustrated in Fig. 2(b) and (c). For example, in ferromagnetic PtCo, the Pt exhibits a $5 \mathrm{~d}$ spin moment of
$0.446 \mu_{\mathrm{B}}$ and an sp-electron contribution of $-0.052 \mu_{\mathrm{B}}$, but in the antiferromagnetic configuration the Pt moment collapses [22]. By contrast, the Co moment is very stable, changing by only $5.6 \%$ when going from ferromagnetism $\left(1.786 \mu_{\mathrm{B}}\right)$ to antiferromagnetism $\left(1.688 \mu_{\mathrm{B}}\right)$.

The spin structures of various $\mathrm{L}_{0}$ alloys with the composition MT ( $\mathrm{M}=\mathrm{Rh}, \mathrm{Pd}, \mathrm{Ir}, \mathrm{Pt} ; \mathrm{T}=\mathrm{Mn}, \mathrm{Fe}, \mathrm{Co}, \mathrm{Ni})$ have recently been investigated by self-consistent spin-polarized LMTO-ASA calculations [19], using a local force theorem and applying a Green-function method [25]. The $J_{i j}$ were calculated perturbatively, Fe and Co favors ferromagnetism, although antiferromagnetic order may be possible in some Fe-containing compounds (Section 3). The Ni compounds are paramagnetic, with the exception of the ferromagnetic NiPd. The manganese-containing alloys are antiferromagnetic, reflecting the well-known general trend towards antiferromagnetism for half-filled $3 \mathrm{~d}$ shells. In addition to the antiferromagnetic interlayer coupling, Mn spins exhibit a strong antiferromagnetic intraplane interaction, as in Fig. 2(c). This indicates that the spin-structure of any given Mn plane is possibly a checkerboard pattern, but a systematic and self-consistent exploration of antiferromagnetic configurations is a challenge for future research.

Note that Heisenberg interactions require stable atomic spin moments. In $\mathrm{L}_{0}$ magnets, this is a good approximation for $3 \mathrm{~d}$ atoms but breaks down completely for the $4 \mathrm{~d} / 5 \mathrm{~d}$ atoms, limiting the applicability of pertubative $J_{i j}$ calculations based on the force theorem. For example, the Heisenberg reversal of an atomic moment may not yield the correct energy, because the switching of an atomic spin (rather than a small-angle perturbation) leads to an adjustment of the oneelectron states, as implied in Fig. 2(b) and (c).

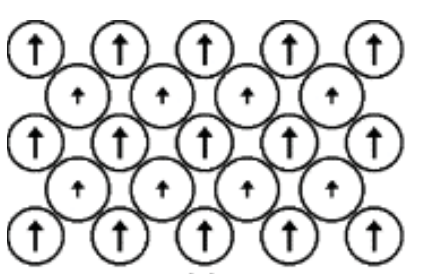

(a)

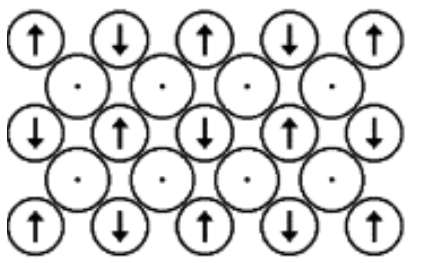

(c)

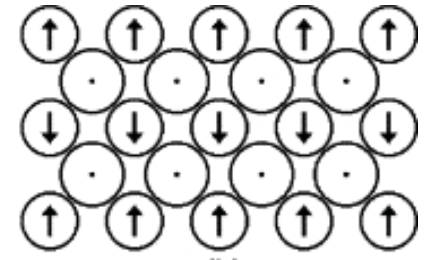

(b)

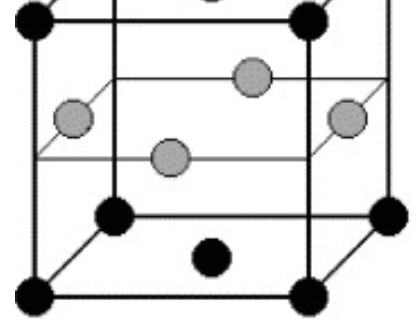

(a) (b)
Fig. 1. Structure of $\mathrm{L}_{0}$-type magnets: (a) equiatomic composition and (b) general composition $\left(\mathrm{ABC}_{2}\right)$.

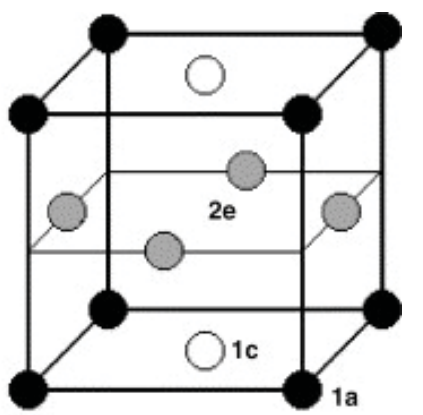

(

Fig. 2. $\mathrm{L} 1_{0}$ spin structures (schematic): (a) ferromagnetism; (b) and (c) antiferromagnetism; (d) the small atoms (those with the large magnetization arrows) are the light transition-metal atoms (3d), as compared to the bigger $4 \mathrm{~d} / 4 \mathrm{f}$ atoms. 


\section{Physical origin of noncollinearity}

There are three basic phenomena leading to noncollinear spin states: (i) competing exchange, (ii) thermal disorder, and (iii) spin-orbit coupling. Noncollinearity due to competing exchange is encountered, for example, in some elemental rare earths (helimagnetism) [26] and depicted in Fig. 1(d). Considering exchange interactions between nearest $\left(J_{0}\right)$ and next-nearest $\left(J^{\prime}\right)$ layers and denoting the magnetization angle of the $n$th transition metal layer by $\theta_{n}$ leads to the total energy

$$
E \sim-J_{0} \sum_{n} \cos \left(\theta_{n+1}-\theta_{n}\right)+J^{\prime} \sum_{n} \cos \left(\theta_{n+2}-\theta_{n}\right)
$$

Putting $\theta_{n+1}=\theta_{n}+\theta^{\prime}$ and minimizing the energy, $\partial E / \partial \theta^{\prime}=0$, yields $\left(J_{0}+4 J^{\prime} \cos \theta^{\prime}\right) \sin \theta^{\prime}=0$. This equation has ferromagnetic $\left(\theta^{\prime}=0\right)$, antiferromagnetic $\left(\theta^{\prime}=\pi\right)$, and helimagnetic $\left(0<\theta^{\prime}<\pi\right)$ solutions. The helimagnetic state occurs for certain negative $J^{\prime}$ values and is characterized by $\theta^{\prime}=\arccos \left(-J_{0} / 4 J^{\prime}\right)$. Note that the modulation wave vector $k \sim 1 / \theta^{\prime}$ is incommensurate with the lattice spacing, in spite of the perfect periodicity expressed in Eq. (1).

Thermal excitations create noncollinearity by randomizing the atomic moments, thereby determining properties such as finite-temperature anisotropy (Section 4) and critical temperature $T_{\mathrm{c}}$. The popular but rough mean-field estimate $T_{\mathrm{c}}=2\langle J\rangle / 3 k_{\mathrm{B}}$, where $\langle J\rangle$ is an averaged exchange, is unable to account for the spatial dispersion of the exchange. In an improved site-resolved or 'lattice' mean-field analysis, the Curie temperature is obtained by diagonalizing an $N \times N$ matrix where $N$ is the number of nonequivalent sites. This approach, originally used to describe multisublattice antiferromagnets [27], can also be used to describe disordered magnets and magnetic nanostructures [9], [28] and [29]. Another aspect of thermal disorder is a reduction of coercivity, by thermally activated magnetization reversal and by decreasing $K_{1}(\mathbf{r})$ [9] and [29].

Chemical disorder further complicates the picture. Concerning the occupancy of the 1a and 1e sites (Fig. 1) by A and $\mathrm{B}$ atoms, there are several scenarios, such in-plane order-disorder transitions, segregation into patches of $\mathrm{A}$ and $\mathrm{B}$ atoms, and various types of interlayer correlations, for example superlattices [30]. Beyond this, interlayer chemical disorder strongly affects the magnetic order [31]. For example, there are first-principle calculations on FePt [17] predicting an antiferromagnetic ground state by a small energy difference of about $0.6 \mathrm{mRy}$ (less than $100 \mathrm{~K}$ ). This is in contrast to experiment and ascribed to the neglect of realstructure imperfections. Fig. 3 illustrates that a relatively small concentration of strongly ferromagnetic Fe bridges may indeed be able to compensate a weak antiferromagnetic bulk interlayer coupling (percolation).

Relativistic effects, such as magnetocrystalline anisotropy, yield noncollinear contributions that are small on an atomic scale but important for mesoscopic phenomena, such as magnetic domains. Expanding the electron energy $m c^{2} \sqrt{1+v^{2} / c^{2}}$ into powers of $v / c$ yields the rest energy $m c^{2}$, the electrostatic energy $m v^{2} / 2$, and the lowest-order relativistic correction $(\alpha / 2)^{2} m v^{2} / 2$ [9] and [32]. Here we have exploited that typical electron velocities in solids are of order $v=\alpha c$, where $\alpha=4 \pi \varepsilon_{0} e^{2} / \hbar c \approx 1 / 137$ is Sommerfeld's finestructure constant [9] and [32]. In real space, relativistic effects are important on length scales of $a_{0} / \alpha=7.2 \mathrm{~nm}$, for example in the context of domains and domain walls [32].

There are several relativistic effects of important in the present context. Anisotropic exchange, $J_{x x, i j} S_{x, i} S_{x, j}+J_{y y, i j}$ $S_{y, i} S_{y, j}+J_{z z, i j} S_{z, i} S_{z, j}$, means that the coupling depends on the spin direction. The corresponding anisotropy is a small relativistic correction to the leading isotropic exchange $\left(J_{x}\right.$ ${ }_{, i j} \approx J_{y, i j} \approx J_{z, i j} \approx J_{i j}$ ) and usually neglected. Magnetocrystalline anisotropy and - in a sense - magnetostatic interactions are small relativistic corrections, too, but unlike the anisotropic exchange they cannot be considered as a small correction to a leading nonrelativistic term. Note that exchange scales as $A k^{2}$, where $A$ is the exchange stiffness, so that $A k^{2} \gg K_{1}$ and $A k^{2} \approx K_{1}$ on atomic and mesoscopic length scales, respectively [9] and [32]. A third type of relativistic corrections is the Dzyaloshinskii-Moriya (DM) interaction $H_{\mathrm{DM}}=-1 / 2 \sum_{i j} \mathbf{D}_{i j} \cdot \mathbf{S}_{i} \times \mathbf{S}_{j}$, where nonzero vectors $\mathbf{D}_{i j}=-\mathbf{D}_{j i}$ reflect the absence of inversion symmetry as a crystalline property or due to disorder. DM interactions are encountered in materials such as $\alpha-\mathrm{Fe}_{2} \mathrm{O}_{3}$, in amorphous magnets [26], in spin glasses [26], and in magnetic nanostructures [9]. They lead to small canting noncollinearities, typically of the order of $0.1^{\circ}$, but the effect may be enhanced for strong disorder and in the vicinity of ferromagnetic-antiferromagnetic transitions.


(b)

Fig. 3. Coupling scenarios in PtFe: (a) perfect $\mathrm{L} 1_{0}$ ordering and (b) imperfect structure where some Fe atoms (black) occupy Pt sites and form FM interlayer bridges. 


\section{Finite-temperature anisotropy}

The leading source of magnetic anisotropy in $\mathrm{L}_{0}$ magnets, of the order of $5 \mathrm{MJ} / \mathrm{m}^{3}$, is magnetocrystalline anisotropy, where the magnetization (spin) talks to the structurally anisotropic environment by spin-orbit coupling. By comparison, the magnetostatic contribution is relatively small, typically of the order of $-0.3 \mathrm{MJ} / \mathrm{m}^{3}$. Magnetocrystalline anisotropy is, essentially, a single-ion property, that is, magnetic anisotropy is realized by embedding the atom in a metallic or nonmetallic crystalline environment [6] and [33]. This must be compared to the popular Néel model [34], where anisotropy is realized by pair interactions. Fig. 4 illustrates the difference. The Néel model requires two interacting magnetic atoms (black), whereas the single-ion or crystal-field model amounts to hopping or crystal-field interactions with atoms that are not necessarily magnetic (white). The principal failure of the Néel model is seen by comparing $\mathrm{Sm}_{2} \mathrm{Fe}_{17}$ and $\mathrm{Sm}_{2} \mathrm{Fe}_{17} \mathrm{~N}_{3}$, where the electronegative nitrogen is nonmagnetic but strongly affects the crystal field and changes the room-temperature anisotropy from $-0.8 \mathrm{MJ} / \mathrm{m}^{3}$ to $8.6 \mathrm{MJ} / \mathrm{m}^{3}[6]$.

The temperature dependence of the anisotropy reflects means that thermal activation affects the crystal-field interaction of the 'black' atom in Fig. 4(b) [6]. In ferromagnetic $\mathrm{L} 1_{0}$ magnets, thermal activation yields some admixture of antiferromagnetic character, thereby reducing the $4 \mathrm{~d} / 5 \mathrm{~d}$ moment and the leading $4 \mathrm{~d} / 5 \mathrm{~d}$ anisotropy contribution. Consider the two-sublattice mean-field Hamiltonian $H=H_{3 \mathrm{~d}}+H_{4 \mathrm{~d} / / \mathrm{d}}+H^{*}$. Here $H_{3 \mathrm{~d}}=-J_{3 \mathrm{~d}} \mathbf{S} \cdot\langle\mathbf{S}\rangle$ is the $3 \mathrm{~d}$ sublattice exchange,

$$
\begin{aligned}
H_{4 \mathrm{~d} / 5 \mathrm{~d}}= & 1 / 2(W-I)\left(m_{z}^{2}+m_{x}^{2}+m_{y}^{2}\right) \\
& -K_{0}\left(m_{z}^{2}-m_{x}^{2} / 2-m_{y}^{2} / 2\right)
\end{aligned}
$$

describes the $4 \mathrm{~d} / 5 \mathrm{~d}$ atoms, and $H^{*}=-J^{*} \mathbf{m} \cdot\left\langle S_{z}\right\rangle$ is the intersublattice interaction. In these equations, $J_{3 \mathrm{~d}}$ and $J^{*}$ are exchange constants, and $\mathbf{S}$ and $\mathbf{m}$ are the $3 \mathrm{~d}$ and $4 \mathrm{~d} / 5 \mathrm{~d}$ moments, respectively. $K_{0}$ is a spin-orbit coupling and crystalfield dependent anisotropy parameter (about $20 \mathrm{MJ} / \mathrm{m}^{3}$ for $\mathrm{PtCo}$ ), and the respective hopping and Stoner parameters $W$ and $I$ determine the $4 \mathrm{~d} / 5 \mathrm{~d}$ spin polarization. The calculation of the free energy is straightforward [22] and yields

$$
K_{1}(T)=K_{1}(0) M_{\mathrm{Co}}^{2}(T) / M_{\mathrm{Co}}^{2}(0)
$$

where $K_{1}(0)=3 K_{0} m^{2} / 2$ and $m=J^{*} S /(W-I)$. Taking into account that $M_{\mathrm{s}} \approx M_{3 \mathrm{~d}}$, Eq. (3) amounts to $K_{1} \sim M_{s}^{m}$ where $m=2$ [22]. By comparison, uniaxial $3 \mathrm{~d}$ magnets, such as $\mathrm{Co}$ and $\mathrm{YCo}_{5}$, exhibit $m=3$ [35], for cubic and noncubic actinide magnets $m=1$ [36], and cubic 3d magnets, such as $\mathrm{Fe}$ and Ni, exhibit $m=10$ [35]. Finally, in rare-earth transition-metal intermetallics the $4 \mathrm{f}$ sublattice anisotropy is largely independent of the $3 \mathrm{~d}$ magnetization [6], so that $m \approx 0$. The exponents $m=2$ and $m=3$ are not very dissimilar [37], but the different physics - the crucial involvement of two sublattices-speaks in favor of $m=2$. Refined calculations by Mryasov et al. [11] have yielded $m=2.08$, amounting to a single-sublattice contribution of the order of $8 \%$. As also pointed out in [11], the reduction of the number of $3 \mathrm{~d}$ neighbors in magnetic nanoparticles has a very similar surface-anisotropy reduction effect.

\section{Micromagnetic description}

On a continuum level, the unit-cell averaged local magnetization $\mathbf{M}(\mathbf{r})$ - and derived properties, such as $H_{\mathrm{c}}$ and $M_{r}$-are obtained by considering micromagnetic energy functionals such as [6], [9] and [38]

$$
E=\int\left[A\left(\nabla\left(\mathbf{M} / M_{\mathrm{s}}\right)\right)^{2}-K_{1}(\mathbf{r}) M_{z}^{2} / M_{\mathrm{s}}^{2}-\mu_{0} \mathbf{M} \cdot \mathbf{H}\right] \mathrm{d} V
$$

Depending on the considered system, additional terms must be added. In lowest order, DM interactions amount to a random field $\sum_{j}\left(D_{i j, y} \mathbf{e}_{x}-D_{i j, x} \mathbf{e}_{y}\right) / 2$ where the summation (or integration) over $j$ includes all atomic neighbors; the resulting structure may be called a "spin colloid". Going beyond the bond-isotropic local expression $\int A\left(\nabla\left(\mathbf{M} / M_{\mathrm{s}}\right)\right)^{2} \mathrm{~d} V$, Heisenberg exchange is described by

$$
E_{\mathrm{ex}}=\iint J\left(\mathbf{r}-\mathbf{r}^{\prime}\right) \mathbf{M}(\mathbf{r}) \cdot \mathbf{M}\left(\mathbf{r}^{\prime}\right) \mathrm{d} V \mathrm{~d} V^{\prime}
$$

Fourier transformation diagonalizes this equation for $J\left(\left|r-\mathbf{r}^{\prime}\right|\right)$ and yields terms of the type $J(\mathbf{k})$, as compared to $A \mathbf{k}^{2}$. Noncollinear or incommensurate spin states then correspond to a minimum of $J(\mathbf{k})$.

For isotropic RKKY systems, the integration in Eq. (5) can be performed analytically and yields a Fourier-transformed interaction $J_{\mathbf{k}}$ that is proportional to the Lindhard screening function $F(k)$. This function [39] reproduces the



(a)

(b)

Fig. 4. Models of magnetic anisotropy: (a) Néel model and (b) crystal-field model. Both models reproduce the correct symmetry, but (b) is physically more adequate for most systems. 
long-wavelength exchange-stiffness approximation $A k^{2}$ but breaks down for $k \sim k_{\mathrm{F}}$. In noncubic materials, $A$ must be replaced by a $3 \times 3$ exchange-stiffness tensor $A_{\mu \nu}$, and the energy is $\sum_{\mu v} \int A_{\mu \nu} \partial \mathbf{M} / \partial x_{\mu} \cdot \partial \mathbf{M} / \partial x_{v} \mathrm{~d} V$. Here $\mu$ and $v$ denote the real-space coordinates $x, y$, and $z$, as contrasted to the relativistic anisotropic exchange $\sum_{\alpha \beta} \int A_{\alpha \beta} \nabla M_{\alpha} \cdot \nabla M_{\beta} \mathrm{d} V$, where $\alpha$ and $\beta$ describe magnetization components.

\section{Discussion and conclusions}

An issue of key importance for the understanding of $\mathrm{L}_{0}$ magnetism is the applicability of atomistic and continuum descriptions. Continuum approaches are able to describe domain-wall motion and magnetization reversal on lengths scales of more than $10 \mathrm{~nm}$ [9] and [12], whereas atomistic first-principle calculations yield increasingly powerful predictions of atomic-scale properties. Similarly, the quantum nature of magnetism is important for very fast processes (above about $10 \mathrm{GHz}$ ), whereas slower magnetization processes can often be treated on a quasi-classical level. Sophisticated equilibrium first-principles calculations can now be used to treat a few hundred nonequivalent atomic sites, and for less sophisticated tight-binding calculations, this number reaches many thousands of atoms. This is sufficient to investigate nanoscale effects at grain boundaries but insufficient to provide a full-scale treatment of phenomena such as the motion and pinning of macroscopic domain walls in particulate thin-film magnets, where about $10^{8}$ atoms must be considered [40].

Micromagnetic model calculations may break down at very small length scales [41], but the effect is relatively small. To investigate the spin structure at sharp atomic grain boundaries [9] and [42], continuum calculations were compared with the corresponding atomistic (layerresolved) $J_{i j}$ summation. Both approximations yield the same behavior, an exponential magnetization decay in the bulk, except for a correction of about $1 \%$ to the penetration depth $\delta_{0}=\left(A / K_{1}\right)^{1 / 2}$. Physically, exchange smoothes magnetization inhomogenities and improves the applicability of continuum theory. However, this does not question the usefulness of atomistic calculations to derive, for example, micromagnetic parameters for atomic structures as those in Fig. 1 and Fig. 3.

In conclusion, we have investigated the magnetism of $\mathrm{L1}_{0}$ magnets on length scales ranging from atomic to macroscopic. In a strict sense, continuum calculations break down on an atomic scale, but noncollinearities due to relativistic interactions (domains and domain walls) are relatively small and smoothed by interatomic exchange. Basically, there is a broad range of phenomena where both approaches can be used. Atomic-scale $\mathrm{L} 1_{0}$ magnetism reflects the two-sublattice nature of the structure. The $3 \mathrm{~d}$ atoms determine the basic spin structure, that is, ferro- or paramagnetic for late $3 \mathrm{~d}$ elements and antiferromagnetic in the mid- dle of the $3 \mathrm{~d}$ series, with a possibility of noncollinear structures, too. The finite-temperature anisotropy of $\mathrm{L}_{0}$ magnets reflect the thermal randomization of the crystal field acting on the $4 d / 5 d$ atoms, accompanied by a collapse of the $4 d / 5 d$ moment.

\section{Acknowledgement}

This research is supported by NSF-MRSEC, AFOSR, the W.M. Keck foundation, and CMRA.

\section{References}

[1] L. Graf and A. Kussmann, Z Phys 36 (1935), p. 544.

[2] W. Jellinghaus, Z Tech Physik 17 (1936), p. 33.

[3] R.M. Bozorth, Ferromagnetism, van Nostrand, Princeton, NJ (1951).

[4] R.A. McCurrie, Ferromagnetic materials-structure and properties, Academic Press, London (1994).

[5] T. Klemmer, D. Hoydick, H. Okumura, B. Zhang and W.A. Soffa, Scripta Metall Mater 33 (1995), p. 1793.

[6] R. Skomski and J.M.D. Coey, Permanent magnetism, Institute of Physics, Bristol (1999).

[7] Y. Shao, M.L. Yan and D.J. Sellmyer, J Appl Phys 93 (2003), p. 8152.

[8] H. Okumura, J. Twisselmann, R.D. McMichael, M.Q. Huang, Y.N. Hsu and D.E. Laughlin et al., J Appl Phys 93 (2003), p. 6528.

[9] R. Skomski, J Phys Condens Matter 15 (2003), p. R841.

[10] D.J. Sellmyer, Nature $\mathbf{4 2 0}$ (2002), p. 374. D.J. Sellmyer, M. Yu, R.A. Thomas, Y. Liu and R.D. Kirby, Phys Low-Dim Struct 1-2 (1998), p. 155.

[11] Mryasov ON, Nowak U, Guslienko K, Chantrell R, in press.

[12] T. Schrefl, J. Fidler and H. Kronmüller, Phys Rev B 49 (1994), p. 6100.

[13] R.F. Sabiryanov and S.S. Jaswal, J Magn Magn Mater 177181 (1998), p. 989.

[14] H. Zeng, R.F. Sabiryanov, O. Mryasov, M.L. Yan, K. Cho and D.J. Sellmyer, Phys Rev B 66 (2002), p. 184425.

[15] K.D. Belashchenko and V.P. Antropov, J Appl Phys 91 (2002), p. 8474.

[16] V.L. Moruzzi and P.M. Marcus, Phys Rev B 48 (1993), p. 16106.

[17] Brown G, Kraczek B, Janotti A, Schulthess TC, Stocks GM, Johnson DD. Phys Rev B 2003;68:052405-1.

[18] G.H.O. Daalderop, P.J. Kelly and M.F.H. Schuurmans In: J.A.C. Bland and B. Heinrich, Editors, Ultrathin magnetic structures I, Springer, Berlin (1994), p. 40.

[19] A. Kashyap, R. Skomski, A.K. Solanki, Y.F. Xu and D.J. Sellmyer, J Appl Phys 95 (2004), p. 7480.

[20] K.D. Belashchenko, J Magn Magn Mater 270 (2004), p. 415. 
[21] P. Ravindran, A. Kjekshus, H. Fjellvag, P. James, L. Nordstrom and B. Johansson et al., Phys Rev B 63 (2001), p. 144409 .

[22] R. Skomski, A. Kashyap and D.J. Sellmyer, IEEE Trans Magn 39 (2003), p. 2917. [23] R. Skomski, Europhys Lett 48 (1999), p. 455.

[24] R. Skomski, R.F. Sabiryanov and S.S. Jaswal, J Appl Phys 85 (2000), p. 5890.

[25] A.I. Liechtenstein, M.I. Katsnelson, V.P. Antropov and V.A. Gubanov, J Magn Magn Mater 67 (1987), p. 65.

[26] K. Moorjani and J.M.D. Coey, Magnetic glasses, Elsevier, Amsterdam (1984).

[27] J.S. Smart, Effective field theories of magnetism, Saunders, Philadephia, PA (1966).

[28] R. Skomski and D.J. Sellmyer, J Appl Phys 87 (2000), p. 4756.

[29] R. Skomski, D. Leslie-Pelecky, R.D. Kirby, A. Kashyap and D.J. Sellmyer, Scripta Mater 48 (2003), p. 857.

[30] Willoughby Sh, MacLaren JM, in press.

[31] T.C. Schulthess, W.H. Butler, G.M. Stocks, S. Maat and G.J. Mankey, J Appl Phys 85 (1999), p. 4842.
[32] R. Skomski, J Magn Magn Mater 272-276 (2004), p. 1476.

[33] C.J. Ballhausen, Ligand field theory, McGraw-Hill, New York, NY (1962).

[34] L. Néel, J Phys Radium 15 (1954), p. 225.

[35] E.R. Callen and H.B. Callen, Phys Rev 129 (1963), p. 578.

[36] R. Skomski, J Appl Phys 91 (2002), p. 8489.

[37] N.H. Hai, N.M. Dempsey and D. Givord, IEEE Trans Magn 39 (2003), p. 2914.

[38] W.F. Brown, Micromagnetics, Wiley, New York, NY (1963).

[39] P. Fulde, Electron correlations in molecules and solids, Springer, Berlin (1991).

[40] J. Zhou, A. Kashyap, Y. Liu, R. Skomski and D.J. Sellmyer, IEEE Trans Magn 40 (2004), p. 2940.

[41] H.R. Hilzinger and H. Kronmüller, Phys Stat Sol (b) 54 (1972), pp. 593-604.

[42] R. Skomski In: M. Ziese and M.J. Thornton, Editors, Spin electronics, Springer, Berlin (2001), p. 204. 